\title{
Office-based ambulatory cervical ripening prior to inpatient induction of labor
}

\author{
There is growing literature to support the use of ambulatory cervical ripening \\ (CR) for low-risk women-initiating CR in the office, sending the patient \\ home to complete the first phase of the CR process, and then admitting her \\ to the labor unit for additional CR or induction of labor
}

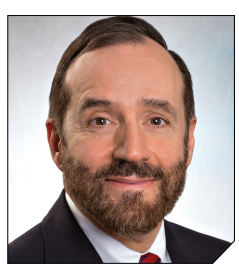

\section{Robert L. Barbieri, MD}

Chair Emeritus, Department of Obstetrics and Gynecology

Interim Chief, Obstetrics

Brigham and Women's Hospital

Kate Macy Ladd Distinguished Professor of Obstetrics,

Gynecology and Reproductive Biology

Harvard Medical School

Boston, Massachusetts ra or women with a Bishop score $\leq 6, \mathrm{CR}$ is an important first step in planned induction of labor (IOL). CR is believed to reduce the length of labor induction and increase the probability of a vaginal delivery. Historically, CR has been undertaken on a labor unit. However, with an increased rate of labor induction, the resources of the modern labor unit are incredibly stressed. Compounding the problem is the nursing shortage caused by the COVID-19 pandemic, which has resulted in staff being unavailable as they recover from a respiratory infection or are quarantined after an exposure. The COVID-19 pandemic also has motivated many patients to avoid the hospital as much as possible.

Office-based ambulatory CR is an alternative to inpatient CR and has the potential to reduce the use of labor unit resources. When CR is initiated in the office, the patient either is sent home overnight to return to the labor unit for IOL in the

doi: 10.12788/obgm.0078 morning or is sent home in the morning to return for IOL in the evening or at night. A secondary benefit of office- and home-based CR is that it may increase patient satisfaction with the process of CR. This editorial summarizes the literature supporting office-based ambulatory CR.

\section{Mechanical methods of CR}

Contemporary mechanical methods of CR include the transcervical insertion of a Foley catheter, Cook double-balloon CR catheter, Dilapan-S, or laminaria. There are many publications reporting the feasibility of office-based ambulatory CR with transcervical balloon catheters and very few publications reporting on the use of Dilapan-S or laminaria for ambulatory CR.

\section{Foley catheter}

Many studies have investigated the effectiveness of transcervical Foley catheter for ambulatory CR. Policiano and colleagues compared the effectiveness of ambulatory versus inpatient Foley catheter CR. ${ }^{1}$ A total of 130 women with a Bishop score $<6$ at $\geq 41$ weeks' gestation were randomly assigned to outpatient or inpatient CR with a transcervical Foley catheter (Covidian Dover Silicon coated latex Foley catheter $16 \mathrm{Fr} / 5.3 \mathrm{~mm}$ diameter). The Foley catheter bulb was distended with $40 \mathrm{~mL}$ of a sterile saline solution. The end of the Foley was taped to the patient's inner thigh. Manual traction was gently applied to the catheter every 6 hours. If the catheter was extruded, the Bishop score was assessed. For a Bishop score $<6$, the patient was given additional inpatient misoprostol ( $25 \mu \mathrm{g}$ vaginally every 4 hours for up to 5 doses). For a Bishop score $\geq 6$, intravenous oxytocin IOL was initiated. At 24 hours if the Foley catheter was still in situ, it was removed. Women were excluded from the study for the following factors: noncephalic presentation, spontaneous labor, hydramnios, nonreassuring cardiotocography (CTG), multiple pregnancy, ruptured membranes, active vaginal bleeding, 
Streptococcus group B infection, and HIV infection. Prostaglandin CR was not used if the woman had a previous cesarean delivery. No prophylactic antibiotics were administered. After placement of the Foley catheter, reassuring CTG was documented prior to sending the patient home.

Outpatient, compared with inpatient, CR resulted in a mean reduction of 10 hours in the time from admission to delivery. The time from insertion of the Foley catheter to delivery in the outpatient group was 38.2 hours, and 44.9 hours for the inpatient group $(P<.01)$. The cesarean delivery rates were similar in both groups-28\% and $38 \%$, respectively. Three cases of chorioamnionitis occurred in each group. These study results support the feasibility of office-based ambulatory $\mathrm{CR}$ with a transcervical Foley.

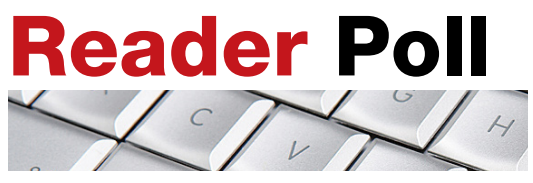

1. Have you used ambulatory cervical ripening in your practice?

2. If so, what is your preferred method for ambulatory

cervical ripening?

- Transcervical Foley catheter

- Transcervical Cook double-balloon CR catheter

- Dilapan-S

- Laminaria

- Oral misoprostol

- Vaginal misoprostol

Tell us at

rbarbieri@mdedge.com.

Please include your name and city and state.
Ausbeck and colleagues randomly assigned 126 nulliparous women with a Bishop score $<5$, at a gestational age ranging from 39 weeks and 0 days through 41 weeks and 6 days, to outpatient overnight CR or inpatient CR with a transcervical Foley catheter. ${ }^{2}$ Breech presentation and multiple gestation pregnancies were excluded from the study. The investigators utilized a 16 French Foley catheter and filled the balloon with $30 \mathrm{~mL}$ of sterile water. The Foley was taped to the woman's inner thigh on slight tension. After placement of the Foley catheter at least 20 minutes of CTG monitoring was performed. The women in the outpatient group were given the contact number for the labor unit and advised that they could take acetaminophen for pain. They were advised that they could stay at home if the Foley catheter was expelled. They were admitted to the labor unit at the time scheduled for their IOL.

The mean time from admission to delivery was reduced by 4.3 hours in the outpatient compared with the inpatient CR group (17.4 vs 21.7 hours; $P<.01$ ). In the outpatient CR group, $22 \%$ of the women were admitted to labor before the time of the scheduled IOL. The cesarean delivery rates were similar in the outpatient and inpatient CR groups (24\% vs $33 \%$, $P=.32$ ). In the outpatient and inpatient groups, chorioamnionitis was diagnosed in $22 \%$ and $13 \%(P=.16)$ of the women. The authors concluded that outpatient CR with a transcervical Foley catheter reduced the time from admission to delivery.

Other research groups also have confirmed the feasibility of outpatient CR with a transcervical Foley catheter. ${ }^{3-5}$

Placement of the Foley catheter can be performed digitally without direct visualization of the cervix or by direct visualization using a vaginal speculum. After placement of the speculum, the cervix is cleansed with a povidone-iodine solution and a sterile ring forceps is used to grasp the catheter and guide it through the cervical os. In one small study, selfreported pain was similar for both digital and direct visualization methods for placement of the balloon catheter. ${ }^{6}$ When using Foley catheter $\mathrm{CR}$, filling the standard Foley catheter balloon with $60 \mathrm{~mL}$ of fluid, rather than 30 to $40 \mathrm{~mL}$ of fluid, is rarely associated with balloon rupture and may result in more effective CR. ${ }^{6,7}$

\section{Double-balloon catheter}

The Cook double-balloon catheter for CR is meant to create pressure on both sides of the cervix, facilitating CR. Studies have reported that the Cook double-balloon catheter can be used for outpatient CR. In one study, 48 women with a low-risk pregnancy, at 37 to 42 weeks' gestation and a Bishop score $<7$ were randomly assigned to outpatient or inpatient double-balloon CR. ${ }^{8}$ Both balloons were filled with 70 to $80 \mathrm{~mL}$ of sterile water. CTG monitoring was performed for 20 minutes before and after balloon placement. The women in the outpatient CR group were instructed to return to the labor unit the next day at $8 \mathrm{AM}$ for IOL or earlier if they had regular uterine contractions, rupture of membranes, or vaginal bleeding. Seven percent of the women in the outpatient group returned to the labor unit before 8 AM. After removal of the balloon catheter, women in the outpatient and inpatient groups needed additional misoprostol CR in $12 \%$ and $13 \%$ of cases, respectively. Outcomes were similar in the two groups, but the study was not powered to identify small differences between the groups. 
In another study of outpatient CR with the Cook double-balloon catheter, 695 women with a Bishop score $<7$, at $\geq 37$ weeks' gestation, were randomly assigned to outpatient CR with a double-balloon catheter or inpatient $\mathrm{CR}$ with dinoprostone $\left(\mathrm{PGE}_{2}\right)$ (2 $\mathrm{mg}$ dinoprostone vaginal gel [Prostin] or dinoprostone $10 \mathrm{mg}$ controlled-release tape (Cervidil). ${ }^{9}$ Women assigned to dinoprostone CR had CTG monitoring prior to commencing $\mathrm{PGE}_{2} \mathrm{CR}$ and at least $30 \mathrm{~min}$ of CTG monitoring after insertion of the vaginal $\mathrm{PGE}_{2}$. Women assigned to balloon CR were not admitted to the hospital. CTG was performed prior to insertion of the balloon. After insertion, the two balloons on the catheter were each filled with $80 \mathrm{~mL}$ of saline. After catheter insertion CTG monitoring was not routinely performed. The women in the double-balloon catheter group returned to the labor unit 12 hours after insertion to initiate IOL. The primary outcome was composite neonatal morbidity and mortality, including admission to a neonatal intensive care unit (NICU), intubation, cardiac compressions, acidemia, hypoxic ischemic encephalopathy, seizure, infection, pulmonary hypertension, stillbirth, or death.

There was no significant difference in the rate of the primary outcome in the catheter versus the $\mathrm{PGE}_{2}$ group (18.6\% and 25.8\%; $P=.07$ ). Admission to the NICU occurred at rates of $12.6 \%$ and $15.5 \%$ in the catheter and $\mathrm{PGE}_{2}$ groups. Umbilical cord arterial $\mathrm{pH}<7.00$ at birth occurred at a rate of $3.5 \%$ in the catheter group and $9.2 \%$ in the $\mathrm{PGE}_{2}$ group. The cesarean delivery rates in the catheter and PGE groups were $32.6 \%$ and $25.8 \%$, respectively $(P=.24)$. The investigators concluded that outpatientCRusing a double-balloon catheter is safe and feasible for nulliparous women.
Two systematic reviews and meta-analyses reported that outcomes were similar when using the Foley or double-balloon catheter for CR. ${ }^{10,11}$ The Cook double-balloon CR kit includes a stylet, which can facilitate passing the catheter through the cervix.

\section{Dilapan-S and laminaria}

There are many published studies using Dilapan-S and laminaria for cervical preparation prior to uterine evacuation. ${ }^{12}$ There are few published studies using Dilapan-S or laminaria for CR prior to IOL. In a pilot study, 21 patients were randomly assigned to outpatient versus inpatient Dilapan-S for CR the night prior to scheduled oxytocin IOL. ${ }^{13}$ The length of time from initiation of oxytocin to delivery in the outpatient and inpatient groups was similar (11 vs 14 hours, respectively). The outpatient compared with the inpatient group had a shorter length of hospitalization until delivery (51 vs 70 hours).

In other studies of Dilapan-S for $\mathrm{CR}$, the patients remained in the hospital once the dilators were inserted. In one small trial, 41 women were randomized to CR with Dilapan-S or laminaria. As many dilators as could be comfortably tolerated by the patient were inserted. ${ }^{14}$ The mean numbers of Dilapan-S and laminaria dilators inserted were 4.3 and 9.7, respectively. The morning after the insertion of the dilators, oxytocin IOL was initiated. The times from initiation of oxytocin to delivery for the women in the Dilapan-S and laminaria groups were 11.6 and 15.5 hours, respectively.

An observational study reported on outcomes with Dilapan-S for CR on inpatients. ${ }^{15}$ In the study 444 women scheduled for IOL at 37 to 40 weeks' gestation, with a mean baseline Bishop score of 2.9, had Dilapan-S placed for approximately 15 hours prior to oxytocin IOL. The mean number of Dilapan-S dilators that were inserted was 3.8. The study protocol prohibited placing more than 5 cervical dilator devices. The mean Bishop score after removal of the dilators was 6.5. The most common adverse effects of Dilapan-S CR were bleeding $(2.7 \%)$ and pain $(0.2 \%)$. The cesarean delivery rate in the cohort was $30.1 \%$. An Apgar score $<7$ at 5 minutes was recorded for 3 newborns. An umbilical artery $\mathrm{pH}$ of $<7.10$ was observed in 8 newborns.

In a randomized trial performed on inpatients, 419 women undergoing CR were assigned to a Foley balloon or Dilapan-S. ${ }^{16}$ The vaginal delivery rates were similar in the groups- $76 \%$ for Foley and $81 \%$ for Dilapan-S. Maternal and neonatal adverse effects were similar between the two groups. Compared with Foley catheter, women assigned to Dilapan-S reported greater satisfaction with their CR experience, more sleep, and more ability to perform daily activities.

\section{Misoprostol and dinoprostone}

Both misoprostol and dinoprostone are effective for outpatient CR. However, a Cochrane systematic review and meta-analysis concluded that balloon CR, compared with prostaglandin $\mathrm{CR}$, is probably associated with a lower risk of uterine hyperstimulation with concerning fetal heart rate changes. ${ }^{17}$ Because misoprostol and dinoprostone occasionally can cause uterine hyperstimulation with fetal heart changes, many experts recommend CTG monitoring both before and after administration of misoprostol or dinoprostone for CR.

In a trial of outpatient versus inpatient vaginal $\mathrm{PGE}_{2} \mathrm{CR}$, 
425 women at 37 to 42 weeks' gestation were assigned randomly to outpatient or inpatient CR. ${ }^{18}$ All women had CTG monitoring for 20 minutes before and after vaginal placement of the $\mathrm{PGE}_{2}$ gel. The $\mathrm{PGE}_{2}$ dose was $2 \mathrm{mg}$ for nulliparous and $1 \mathrm{mg}$ for parous women. The cesarean delivery rates were similar in the outpatient and inpatient groups-22.3\% and $22.9 \%$, respectively. Among the women randomized to outpatient CR, 27 women (13\%) could not be discharged home after administration of the vaginal $\mathrm{PGE}_{2}$ because of frequent uterine contractions or an abnormal fetal heart rate pattern. In addition, 64 women $(30 \%)$ in the outpatient group returned to the hospital before scheduled induction because of frequent contractions. Maternal and neonatal complications were similar in the two groups. The investigators concluded that, at the dose and route of prostaglandin utilized in this study, the resultant rates of abnormal fetal heart rate pattern and frequent contractions might reduce the clinical utility of outpatient vaginal prostaglandin CR.

Another study also reported a greater rate of uterine tachysystole with vaginal $\mathrm{PGE}_{2}$ compared with a Foley catheter for CR (9\% vs 0\%). ${ }^{19}$ In a Cochrane systematic review of vaginal prostaglandin for CR, compared with placebo, vaginal prostaglandins were associated with a significantly greater rate of uterine hyperstimulation with fetal heart rate changes $(4.8 \%$ vs $1.0 \%){ }^{20}$ Other studies also reported the feasibility of outpatient CR with vaginal prostaglandin. ${ }^{21,22}$

Both oral and vaginal misoprostol have been utilized for outpatient CR. In one study, 87 women with singleton pregnancy at 40 to 42 weeks' gestation with a Bishop score $<6$ were randomized to outpatient CR with oral misoprostol $(100 \mu \mathrm{g})$ or placebo. ${ }^{23}$ Following administration of the oral misoprostol, the women had 2 hours of CTG monitoring. The treatment was repeated daily for up to 3 days if there was no change in the cervix. If labor occurred, the patient was admitted to the labor unit for oxytocin IOL. The times from first dose of misoprostol or placebo to delivery were 46 and 84 hours $(P<.001)$, respectively.

In another study, 49 women $\geq 40$ weeks' gestation with a Bishop score $<5$ were randomly assigned to receive outpatient oral misoprostol $25 \mu \mathrm{g}$ or $50 \mu \mathrm{g} .{ }^{24}$ The dose could be repeated every 3 days over 9 days if ripening or labor had not been achieved. The women had CTG before administration of oral misoprostol. After the misoprostol dose, they had 2 hours of CTG monitoring. The number of doses received by the women assigned to the $50 \mu \mathrm{g}$ group were $83 \%, 13 \%$, and $4 \%$ for 1,2 , and 3 doses, respectively. The number of doses received by the women assigned to the $25 \mu \mathrm{g}$ group were $58 \%, 26 \%$, and $16 \%$ for 1,2 , and 3 doses, respectively. The mean intervals from initiation of $\mathrm{CR}$ to delivery in the $25 \mu \mathrm{g}$ and the $50 \mu \mathrm{g}$ groups were 3.9 and 2.5 days, respectively. The investigators reported no maternal or newborn adverse events, although the study was not powered to detect infrequent events.

Many studies have reported on the feasibility of outpatient CR with vaginal misoprostol. ${ }^{25-30}$ In one study, 77 women at 40 weeks' gestation and a Bishop score $\leq 8$ were randomized to a single dose of vaginal misoprostol $25 \mu \mathrm{g}$ or gentle cervical examination (control). ${ }^{25}$ The women had 1 hour of CTG monitoring after the intervention. If they had regular contractions they were admitted to the birthing unit. If they had no regular contractions they were discharged home. For nulliparous women, the time from intervention to delivery in the misoprostol group was 4.9 days, and 8.1 days in the control group. For parous women, the times from intervention to delivery in the two groups were 3.8 and 6.9 days, respectively.

\section{Inclusion and exclusion criteria for outpatient CR}

Outpatient CR should be limited to low-risk women with a singleton gestation, who have reliable access to transportation from home to the labor unit and have a clear understanding of the instructions for outpatient CR. Patient characteristics that may be utilized to offer officebased CR include:

- singleton pregnancy at 39 weeks' and 0 days' gestation through 40 weeks' and 6 days' gestation

- cephalic presentation

- Bishop score $\leq 6$.

Women who should be excluded from outpatient CR include those with:

- contraindications to vaginal delivery

- fetal growth restriction

- abnormal umbilical artery Doppler results

- oligo- or polyhydramnios

- multiple gestation

- major fetal anomaly

- recent nonreactive fetal heart rate tracing

- maternal report of decreased fetal movement

- abnormal biophysical profile

- prior cesarean delivery

- recent vaginal bleeding

- gestational diabetes requiring medication treatment

- significant hypertension.

Practices should establish their own inclusion and exclusion criteria for ambulatory CR. 


\section{Safety of office-based ambulatory CR among low-risk women}

Safety is a complex concept with experts often disagreeing on what level of safety is required to accept a new medical procedure. Establishing the safety of office-based ambulatory $\mathrm{CR}$ among low-risk women would require a very large cohort or randomized studies with at least a thousand participants. Only a few large studies focused on the safety of CR have been reported. Sciscione and colleagues reported a large observational study of inpatient transcervical Foley catheter for CR involving 1,905 women. ${ }^{31}$ They reported no adverse outcomes among term, singleton, uncomplicated pregnancies. They calculated that the $95 \%$ confidence interval (CI) for an adverse event was between $0.0 \%$ and $0.2 \%$. In a meta-analysis of 26 studies including 5,563 women, the risk of chorioamnionitis during IOL was equivalent with pre-IOL Foley catheter CR (7.2\%) or prostaglandin CR $(7.2 \%)$ (relative risk, 0.96; 95\% CI, 0.66-1.38). ${ }^{32}$
Two systematic reviews have reported that, compared with balloon $\mathrm{CR}$, misoprostol $\mathrm{CR}$ is associated with an increased risk of uterine tachysystole. ${ }^{33-34}$ In a large retrospective study, compared with inpatient CR, outpatient CR with dinoprostone vaginal insert was not associated with an increased risk of newborn admission to the neonatal intensive care unit or a low Apgar score at 5 minutes after birth. ${ }^{35}$

\section{Will you consider office-based CR in your obstetric practice?}

As reviewed in this editorial, evolving data suggest that it is feasible to initiate CR in the office ambulatory setting prior to admission to the labor unit for additional CR or IOL. Many women prefer to complete CR at home after initiation in the office, rather than have CR in a labor unit or hospital setting. ${ }^{36}$ The transcervical balloon catheter has the most published data supporting the feasibility of ambulatory CR. Compared with misoprostol, the transcervical balloon catheter is associated with a low rate of uterine tachysystole. It may be a preferred method for outpatient CR. If placement of a transcervical balloon catheter is challenging, for example when the patient has a tightly closed cervix, oral misoprostol ambulatory CR may be an option if CTG monitoring is available in the office.

During the COVID pandemic, many in-person office visits have transitioned to virtual visits with the patient in their home. Historically, most cases of CR have been performed on labor and delivery units. It may be time for your practice to consider office-based ambulatory CR for low-risk women planning an IOL. Office-based ambulatory CR is a win for labor nurses who generally prefer to manage laboring patients rather than patients undergoing prolonged inhospital CR. Outpatient CR is also a win for low-risk patients who prefer to be at home rather than in a labor unit.
References

1. Policiano C, Pimenta M, Martins D, et al. Outpatient versus inpatient cervix priming with Foley catheter: a randomized trial. Eur J Obstet Gynecol Repro Biol. 2017;210:1-6.

2. Ausbeck EB, Jauk VC, Xue Y, et al. Outpatient Foley catheter for induction of labor in nulliparous women. Obstet Gynecol. 2020;136:597-606.

3. Wilkinson C, Adelson P, Turnbull D. A comparison of inpatient with outpatient balloon catheter cervical ripening: a pilot randomized controlled trial. BMC Pregnancy Childbirth. 2015;15:126.

4. Sciscione AC, Muench M, Pollock M, et al. Transcervical Foley catheter for preinduction cervical ripening in an outpatient versus inpatient setting. Obstet Gynecol. 2001;98:751-756.

5. Henry A, Madan A, Reid R, et al. Outpatient Foley catheter versus inpatient prostaglandin E2 gel for induction of labour: a randomised trial. $B M C$ Pregnancy Childbirth. 2013;13:25.

6. Kuhlmann MJ, Spencer N, Garcia-Jasso C, et al. Foley bulb insertion by blind placement compared with direct visualization. Obstet Gynecol. 2021;137:139-145.

7. Delaney S, Shaffer BL, Chen YW, et al. Labor induction with a Foley balloon inflated to 30 $\mathrm{mL}$ compared with $60 \mathrm{~mL}$. Obstet Gynecol. 2015;115:1239-1245.
8. Wilkinson C, Adelson P, Turnbull D. A comparison of inpatient with outpatient balloon catheter cervical ripening: a pilot randomized controlled trial. BMC Pregnancy Childbirth. 2015;15:126.

9. Beckmann M, Gibbons K, Flenady V, et al. Induction of labor using prostaglandin $\mathrm{E}_{2}$ as an inpatient versus balloon catheter as an outpatient: a multicenter randomised controlled trial. BJOG. 2020;127:571-579.

10. Liu X, Wang Y, Zhange F, et al. Double- versus single-balloon catheters for labour induction and cervical ripening: a meta-analysis. BMC Pregnancy Childbirth. 2019;19:358.

11. Yang F, Huan S, Long Y, et al. Double-balloon versus single-balloon catheter for cervical ripening and labor induction: a systematic review and meta-analysis. J Obstet Gynaecol Res. 2018;44: 27-34.

12. Goldberg AB, Fortin JA, Drey EA, et al. Cervical preparation before dilation and evacuation using adjunctive misoprostol and mifepristone compared with overnight osmotic dilators alone: a randomized controlled trial. Obstet Gynecol. 2015;126:599-609.

13. 1Upadhyaya NB, Childs KD, Neiger R, et al. Ambulatory cervical ripening in term pregnancy. J Reprod Med. 1999;44:363-366.
14. Blumenthal PD, Rmanauskas R. Randomized trial of Dilapan and Laminaria as cervical ripening agents before induction of labor. Obstet Gynecol. 1990;75:365-368.

15. Gupta J, Chodankar R, Baev O, et al. Synthetic osmotic dilators in the induction of labour-an international multicenter observational study. Eur J Obstet Gynecol Repro Biol. 2018;229:70-75.

16. Saad AF, Villarreal J, Eid J, et al. A randomized controlled trial of Dilapan-S vs Foley balloon for preinduction cervical ripening (DILAFOL trial). $\mathrm{Am} J$ Obstet Gynecol. 2019;220:275.e1-e9.

17. de Vaan MD, Eikleder MLT, Jozwiak M, et al Mechanical methods for induction of labour Cochrane Database Syst Rev. 2019;CD001233.

18. Wilkinson $C$, Bryce $R$, Adelson $P$, et al. A randomized controlled trial of outpatient compared with inpatient cervical ripening with prostaglandin $\mathrm{E}_{2}$ (OPRA study). BJOG. 2015;122:94-104.

19. Blair R, Harvey MA, Pudwell J, et al. Retrospective comparison of $\mathrm{PGE}_{2}$ vaginal insert and Foley catheter for outpatient cervical ripening. J Obstet Gynaecol Can. 2020;42:1103-1110.

20. Thomas J, Fairclough A, Kavanagh J, et al. Vaginal prostaglandin (PGE2 or PGF2alpha) for induction of labour at term. Cochrane Database Syst Rev 2014;CD003101. 
21. O'Brien JM, Mercer BM, Cleary NT, et al. Efficacy of outpatient induction with low-dose intravaginal prostaglandin E2: a randomized, doubleblind, placebo controlled trial. Am J Obstet Gynecol. 1995;173:1855-1859.

22. Biem SR, Turnell RW, Olatunbosun $\mathrm{O}$, et al. A randomized controlled trial of outpatient versus inpatient labour induction with vaginal controlled-release prostaglandin-E2: effectiveness and satisfaction. J Obstet Gynaecol Can. 2003;25:23-31.

23. Gaffaney CA, Saul LL, Rumney PJ, et al. Outpatient oral misoprostol for prolonged pregnancies: a pilot investigation. Am J Perinatol. 2009;26: 673-677.

24. Kipikasa JH, Adair CD, Williamson J, et al. Use of misoprostol on an outpatient basis for postdate pregnancy. Int J Gynaecol Obstet. 2005;88:108-111.

25. Oboro VO, Tabowei TO. Outpatient misoprostol cervical ripening without subsequent induction of labor to prevent post-term pregnancy. Acta Obstet Gynecol Scand. 2005;84:628-631.
26. Stitely ML, Browning J, Fowler M, et al. Outpatient cervical ripening with intravaginal misoprostol. Obstet Gynecol. 2000;96:684-688.

27. McKenna DS, Ester JB, Proffitt M, et al. Misoprostol outpatient cervical ripening without subsequent induction of labor: a randomized trial. Obstet Gynecol. 2004;104:579-584.

28. PonMalar J, Benjamin SJ, Abraham A, et al. Randomized double-blind placebo controlled study of preinduction cervical priming with $25 \mu \mathrm{g}$ of misoprostol in the outpatient setting to prevent formal induction of labor. Arch Gynecol Obstet. 2017;295:33-38.

29. Chang DW, Velazquez MD, Colyer M, et al. Vaginal misoprostol for cervical ripening at term: comparison of outpatient vs inpatient administration. Obstet Gynecol Surv. 2006;61:167-168.

30. Meyer M, Pflum J, Howard D. Outpatient misoprostol compared with dinoprostone gel for preinduction cervical ripening: a randomized controlled trial. Obstet Gynecol. 2005;105:466-472.

31. Sciscione AC, Bedder CL, Hoffman MK, et al.
The timing of adverse events with Foley catheter preinduction cervical ripening; implications for outpatient use. Am J Perinatol. 2014;31:781-786.

32. McMaster K, Sanchez-Ramos L, Kaunitz AM. Evaluation of a transcervical Foley catheter as a source of infection. Obstet Gynecol. 2015;126:539-551.

33. Fox NS, Saltzman DH, Roman AS, et al. Intravaginal misoprostol versus Foley catheter for labour induction: a meta-analysis. BJOG. 2011;118: 647-654.

34. Hofmeyr GJ, Gulmezoglu AM, Pileggi C. Vaginal misoprostol for cervical ripening and induction of labour. Cochrane Database Syst Rev. 2010:CD000941.

35. Salvador SC, Simpson ML, Cundiff GW. Dinoprostone vaginal insert for labour induction: a comparison of outpatient and inpatient settings. J Obstet Gynaecol Can. 2009;31:1028-1034.

36. Sutton C, Harding J, Griffin C. Patient attitudes towards outpatient cervical ripening prior to induction of labour at an Australian tertiary hospital. J Obstet Gynaecol. 2016;36:921-928. 